\title{
Physics of hypercumulation: jet formation in shaped charge and ablatively-driven implosion of hollow cones
}

\author{
I. V. Minin*, O. V. Minin \\ Siberian State Academy of Geodesy, Plahotnogo 10, Novosibirsk, 630108, Russia \\ *E-mail address: Prof.minin@gmail.com
}

\begin{abstract}
In this letter we suggest a new approach to the physical principles for hypercumulative plasma jet formation. This new approach leads to several new results which are of fundamental importance. The simulation results of hypercumulative plasma jet are discussed. It has been shown that the increase of the plasma jet speed in the suggested configuration is $25-30 \%$ and the increase of jet pulse is more than 90 times which are not achievable in the classical cumulation.
\end{abstract}

Keywords: Ablation; Plasma Jet; Cumulation; Hypercumulation

\section{INTRODUCTION}

Up today the outstanding issue of how collapsing nebulae are able to launch highly collimated beams of matter. This area has been studied through observations and by numerical simulations for many years. But the problem is open. To our opinion well characterized quantitative experiments will play a decisive role in resolving a number of outstanding scientific issues. The understandings of the physics governing the behavior of astrophysical objects via scaled laboratory experiments, combined with computer modeling are perspective.

The properties of plasma jet are determinate by the jet formation mechanism. Several problematics concerning plasma jet formation at initial time can be study numerically in order to provide useful data for astrophysical models. As it was mentioned at [2] the phenomena associated with astrophysical jets, aside from mechanisms of their initial formation, include the morphology of the jet as it bores through an ambient medium, the nature of instabilities that could disrupt the jet's coherence, the mechanisms preserving the jet collimation, the efficiency with which the ambient gas is entrained in the jet, and the the behavior of a jet in a magnetized environment.

The problem of simulating plasma jet from the point of view of extending the applications to simulating flows with temperature-dependent diffusion parameters (viscosity and diffusivity), interaction with and investigation of condensed matter properties, investigations of shock waves and equation of state at extreme high conditions in condensed matter, for synthesis of a new materials or welding new composite materials by interaction of plasma jet with condensed natter target and so on are important. On the other hand the 
problem of plasma accelerators development, producing jets with high kinetic energy (i.e. mass and speed) has its own fundamental and application significance.

In this paper we report the first simulation efforts to possibility of creation of laboratory so-called hypercumulative jet from hollow cones collapsed onto their axes by pressure generated via laser ablation of their outer surfaces with parameters of jet which are not achievable in the classical cumulation.

The organization of this paper is as follows. In the first section, we introduce the brief theory review on cumulative plasma jet formation mechanism. The second section contains a short review of simulation results and discussion. Finally, in the last section we complete the conclusion of the work.

\section{BRIEF THEORY}

The methods of cumulative plasma jet creation by laser beam action on a conically shaped thin metallic foil were studied in axisymmetrical [1-3] and flat [4-5] geometry of target. While the theoretical predictions concerning the plasma jet parameters (jet velocity, collimation and plasma density) were rather promising, relative small maximum velocities were obtained in numerical simulations carried out for $\mathrm{Al}$ and $\mathrm{Au}$ jets [1-5].

\section{1. Brief theory of classical cumulative jet formation}

The plasma cumulative jets were formed by a classical hydrodynamic mechanism [1]. As it well known hydrodynamic theory by G. Taylor, G. Birkhoff [6] and M. Lavrentyev [7] promotes first approximation for the analytical solution of the problem provided that jet forming is managed by steady outflow laws. In experiments mentioned above, cones and wedges were imploded onto their axes by pressure generated via laser ablation of their outer surfaces and were based on classical hydrodynamic theory of cumulative jet.

The main idea of the researches at [1-4] was as follows. For classical shaped charge conditions and geometry, relative jet velocity Vjet and mass Mjet respectively are related to the apex half-angle $\alpha$ as [6-8]

$$
\operatorname{Vjet} \sim \cot (\alpha / 2), \quad \operatorname{Mjet} \sim \sin 2(\alpha / 1)
$$

Formulae (1) predict that very high jet velocity can be achieved at very small angles $\alpha$. From the hydrodynamic theory it is followed that compressibility imposes a lower limit on $\alpha$ and reduces the mass of a jet in comparison with Eqs.(1).It is important that at angles smaller than this lower limit, i.e., $\alpha<\alpha$ crit, the cumulative jet is not produced [5-7]. In an ideal gas and wedge geometry, classical theory predict $\alpha$ crit $=\sin -1(1 / \gamma)[6-8]$ where $\gamma$ is the adiabatic exponent. For $\gamma=5 / 3$ the corresponding apex angle are relative large $2 \alpha \mathrm{crit}=74^{\circ}$ [2].

But the application of jet formation criterion given by formulas (1) to the general nonstationary case and to the case of axial symmetry flows was not supported by precise theoretical results. The theory of cumulative charge functioning has formed rather certain vision of the nature of liner jet-less collapsing in a cumulative hole and forming dispersed cumulative jets. Works [6-8] were of first importance in forming this vision.

It is important that work by Walsh and co-authors published in mid-50s dealt with steady (continuous) symmetrical collision of plain supersonic jets and made an important adjunct to the hydrodynamic theory of cumulation. Formation criterion for the cumulative jet promoted there in was later made a jet formation criterion for the whole nomenclature of 
cumulative charges, non-stationary and axis-symmetrical cases inclusive. Doubts were sometimes expressed about its applicability to the axis-symmetrical case, but they practically did not shake its "authority", as, in practice, the conditions of compact cumulative jet formation were of interest. These conditions need more strict requirements than those offered by Walsh. Simply stated, the implementation of these conditions needs subsonic speeds of jet collision. Necessary exactly conditions of the cumulative jet absence at non-stationary collapse of the cumulative hole are specified at [9]. It has been shown that Walsh criterion is inapplicable for the axis symmetry case. In axis symmetry case, the flow with the shock wave attached to the axis of symmetry shall never realize, which means cumulative jets form at all times. Depending on the speed and angler of collision, solid, partially dispersed or totally dispersed jets (macro-particles flow) may form. So the conclusions about "cumulative jet" limiting velocity and its parameters made at [1-3] are not valid in common case.

Moreover, the main processes that proceed during generation of shaped-charge jet are described by the M. A. Lavrentyev-Birkhoff (the USSR-USA) theory mentioned above as a model of plane stationary impingement of incompressible fluid jets at angles less than 180 degrees. It is a well-known fact confirmed by almost 100 -year practice of designing shaped charges.

\section{2. Brief theory of hypercumulative jet formation} observed:

In the hypercumulative regime of jet formation offered at [10-15] new qualities are

1) The weight of a shaped-charge jet becomes greater than the weight of a slug up to almost full absence of slug.

2) At the jet impingement angles greater than 180 degrees, the jet and slug have changed their places.

3) Velocity of a massive jet is high, while that of a thin slug is low.

It could be noted that direct creation of shaped charges with pointed-nose lining that use movement of materials at angles greater than 180 degrees is impossible within a classical shaped-charge effect.

Creation of a required increased axial velocity of a shaped-charge jet at the expense of energy of an additional body before impingement of lining elements on the charge symmetry axis and during jet formation allows overcoming this limitation. Now, shaped-charge explosion obtains the possibility of creating high maximum jet velocities subject to the required explosive energy, geometrical dimensions and relevant structures that enable using explosion energy to create the required axial $\mathrm{V}_{\mathrm{z}}$ velocities and radial $\mathrm{V}_{\mathrm{r}}$ velocities of lining elements. This allows formation of a super velocity shaped-charge jet without its disintegration. If additional $\mathrm{V}_{\mathrm{z}}$ velocity is small, the shaped-charge flow mode goes into classical domain creating a thin jet and thick massive stamp.

Let us consider a similar model problem. Let an axially symmetrical jet of compressible of ideal fluid with aluminium properties impinge on a copper barrier that is also made of compressible ideal fluid. A non-stationary axially symmetrical problem is considered. Fig. 1 shows formulation of the problem. Velocity of aluminium liquid jet $\left(\mathrm{V}_{\mathrm{z}}\right)$ is $-2.6 \mathrm{~km} / \mathrm{s}$, and radial velocity is $-1.5 \mathrm{~km} / \mathrm{s}$. Velocity of the movable copper barrier is $0.6 \mathrm{~km} / \mathrm{s}$. 


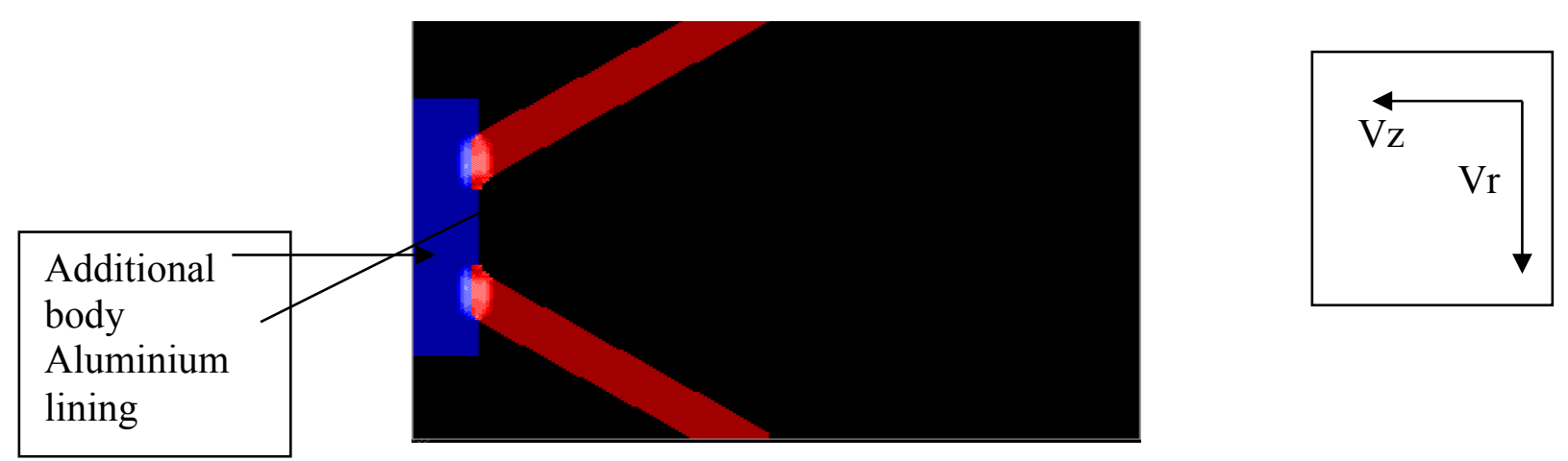

Fig. 1. Formulation of the problem

Table. 1. Hypercumulative jet formation simulation

\begin{tabular}{|l|l|}
\hline $1.2 \mu \mathrm{s}$ & \\
\hline $1.6 \mu \mathrm{s}$ & \\
\hline $2.0 \mu \mathrm{s}$ & \\
\hline
\end{tabular}



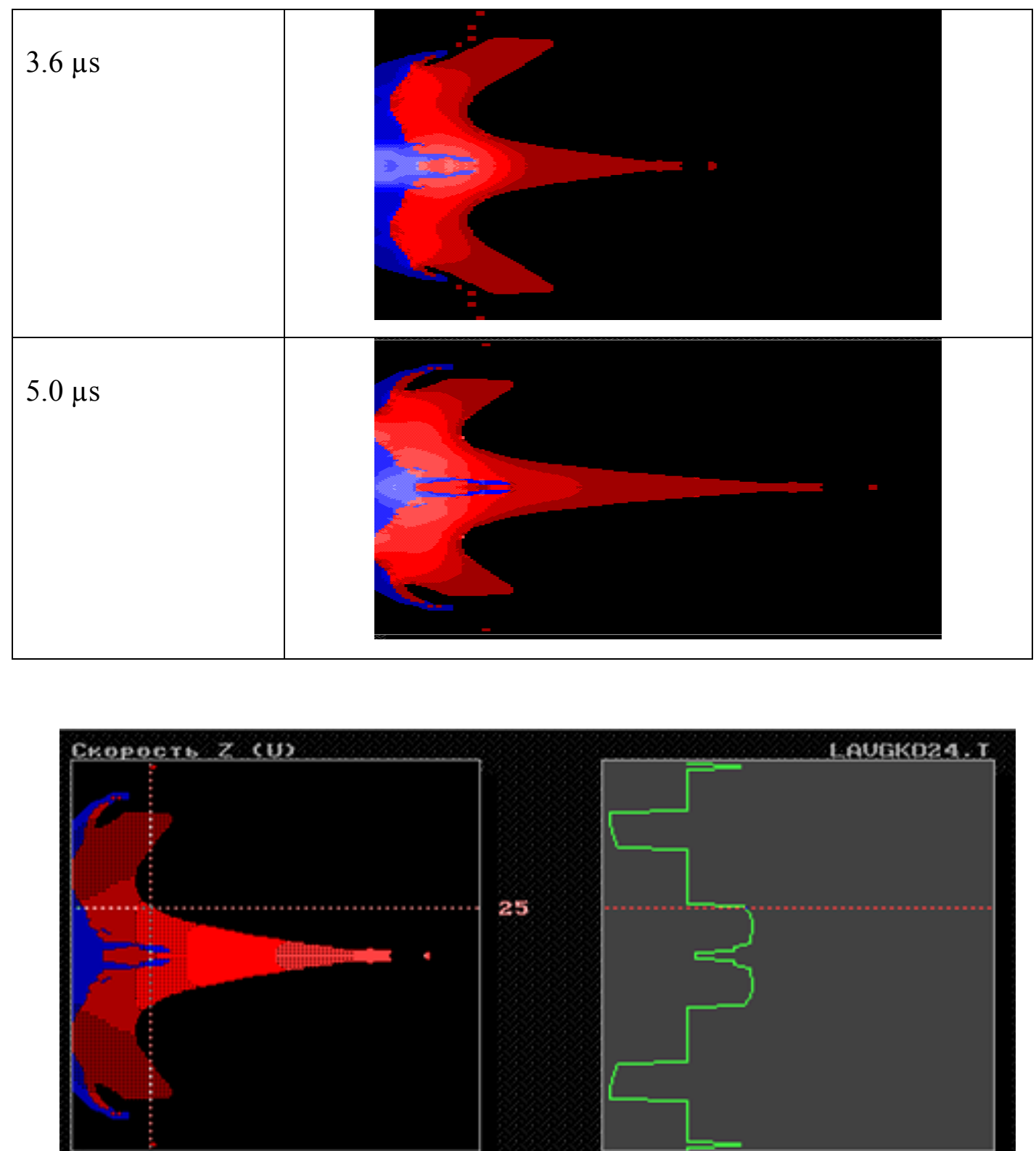

LAUGKO24.T

39
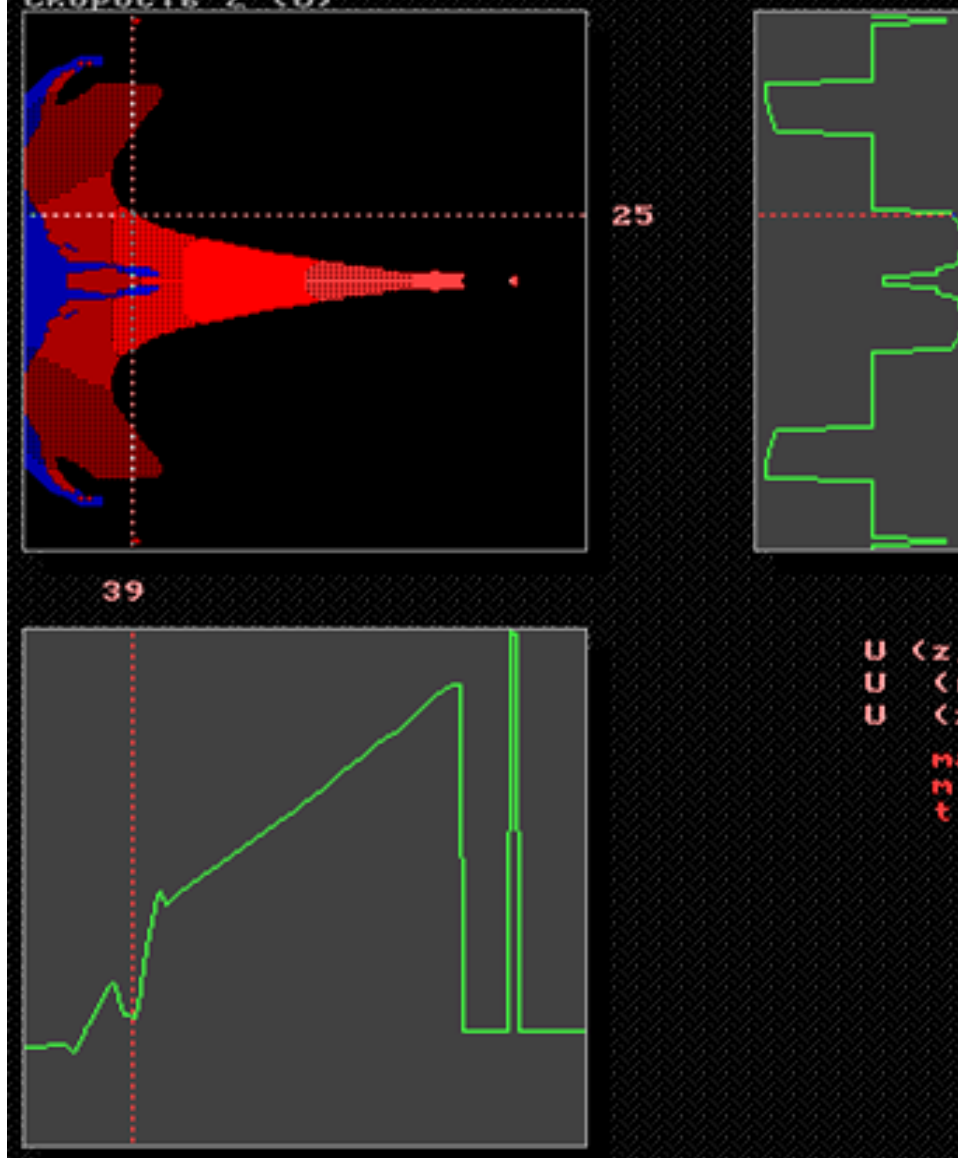

$u(z, r)=1.986 e+00$

U $(r)=1.986 \theta+00$

$u(z)=2.7779-01$

$\max =1 \cdot 0056+01$

min $=-2.950$ - 00

$t$ ine $=5.0000+00$

Fig. 2. The flows of materials at the $5 \mu$ s are shown. Vz velocity isolines and its diagrams in crosssections are shown by the marker. 
Table 1 demonstrates subsequent development of the process with formation of a powerful shaped-charge jet and a thin stamp jet, while the pictures show pressure isolevels at the relevant points of time. Material spalling from the jet head took place in the shaped-charge jet being formed.

As follows from the experiments presented in Table 1, the jet impingement level on the symmetry axis of the problem exceeds 180 degrees. Maximum velocity of the shaped-charge jet being formed is $8.7 \mathrm{~km} / \mathrm{s}$, and, as this takes place, jet material partially breaks away from the jet head with a velocity of approximately $10 \mathrm{~km} / \mathrm{s}$ (Fig. 2). The formed shaped-charge jet diameter has become considerably greater than the stamp diameter.

In our opinion, this problem clearly demonstrates one of the principles of lining collapse at angles exceeding 180 degrees. This is accomplished by placement of a "mirror" in the way of conical jets and "reflection" of their impingement on the symmetry axis. Such an upgraded analytical model can in fact be created.

\section{3. Verification of hypercumulative jet formation principle}

We have developed the simple prototype of shaped charge to explain the hypercumulative jet formation principle. The desing of shaped charge is shown in the Figure 3.

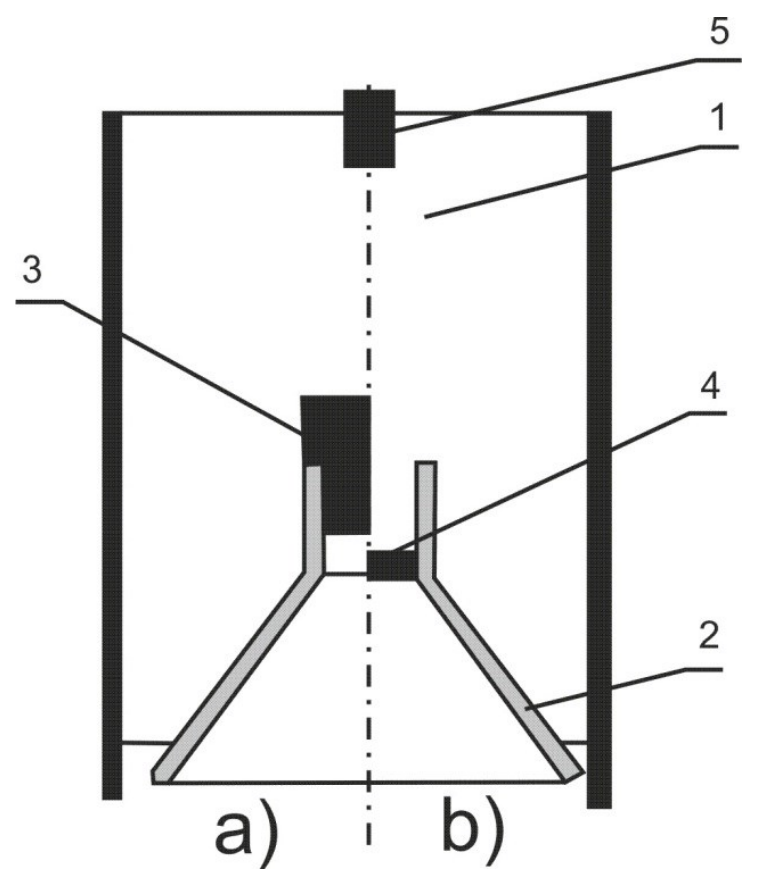

Fig. 3. Design of the classical (a) and hypercumulative (b) shaped charge: 1 - explosive, 2 - aluminum liner (cone with 42 degree), 3 - steel, 4 - cu flat plate, 5 - exploder.

It is clear from the Fig. 4 that hypercumulative jet formation principle allows increasing the diameter of the jet (up to 2 times) and tip velocity of jet (from $7.5 \mathrm{~km} / \mathrm{sec}$ for classical jet to $9.2 \mathrm{~km} / \mathrm{sec}$ for hypercumulative).

Below the application of hypercumulation regime of jet formation to ablatively-driven implosion of hollow cone are described. 


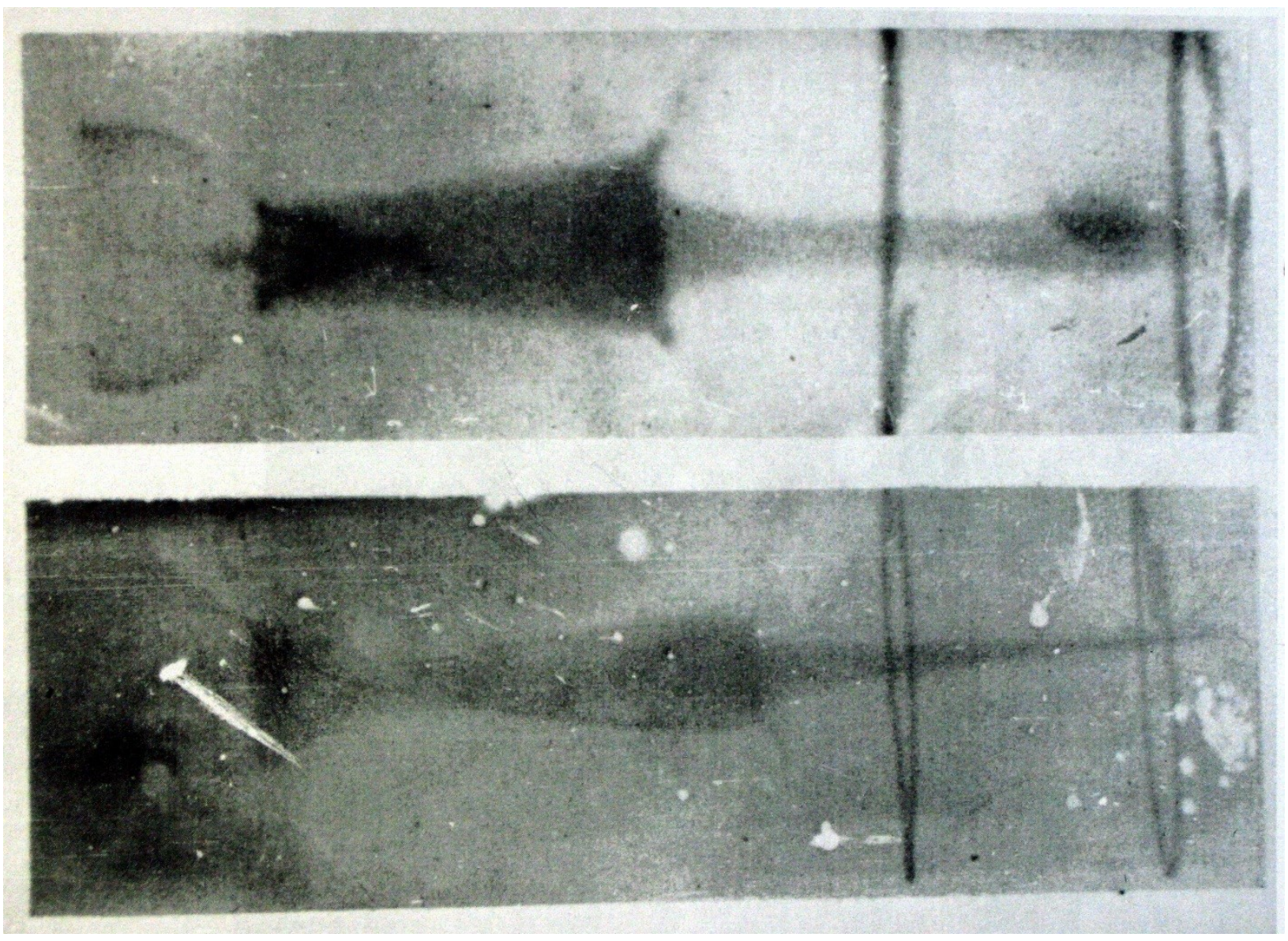

Fig. 4. X-ray of shaped charge jet: classical (top, Fig. 3a) and hypercumulative (lower, Fig. 3b).

\section{SIMULATION RESULTS OF HYPERCUMULATIVE PLASMA JET}

One novel way of increasing the pulse and speed of the cumulative plasma jet by a hydrodynamic mechanism is in realization of the suggested principle of the hypercumulative charges [10-13]. In this case (in simplify geometry) the target has a form of truncated cone with, for example, a flat plate in its apex (Fig. 5). As the pressure pulse acts upon the external surface of the cone the walls of the latter begin to move normally to the surface. The flat (and in some cases profiled) plate behaves like a piston and compresses the material of the side wall of the target collapsing to the symmetry axis. Such mechanism of the jet formation is described with problem of the jet collapse without a point of full breaking with the formation of inner zone of the constant pressure stagnation zone.

Such problem has no the only one solution as the pressure within this zone or the same as the size of such a zone is a free parameter. The presence of the stagnation zone has a smoothing influence upon the evolution of different kinds of disturbances at the jet formation. The realization of the hypercumulation principle allows increasing both the speed and the pulse of the formed jet essentially. More detailed analysis of the analytical model of nonstationary formation of the jet shows that at the propagation of jet not in vacuum but in the media with a low coefficient of resistance, as mentioned above, there is the effect of some increase of the jet speed. And this medium as preliminary numerical experiments show makes a stabilization action on the radial expansion of the jet [11]. 


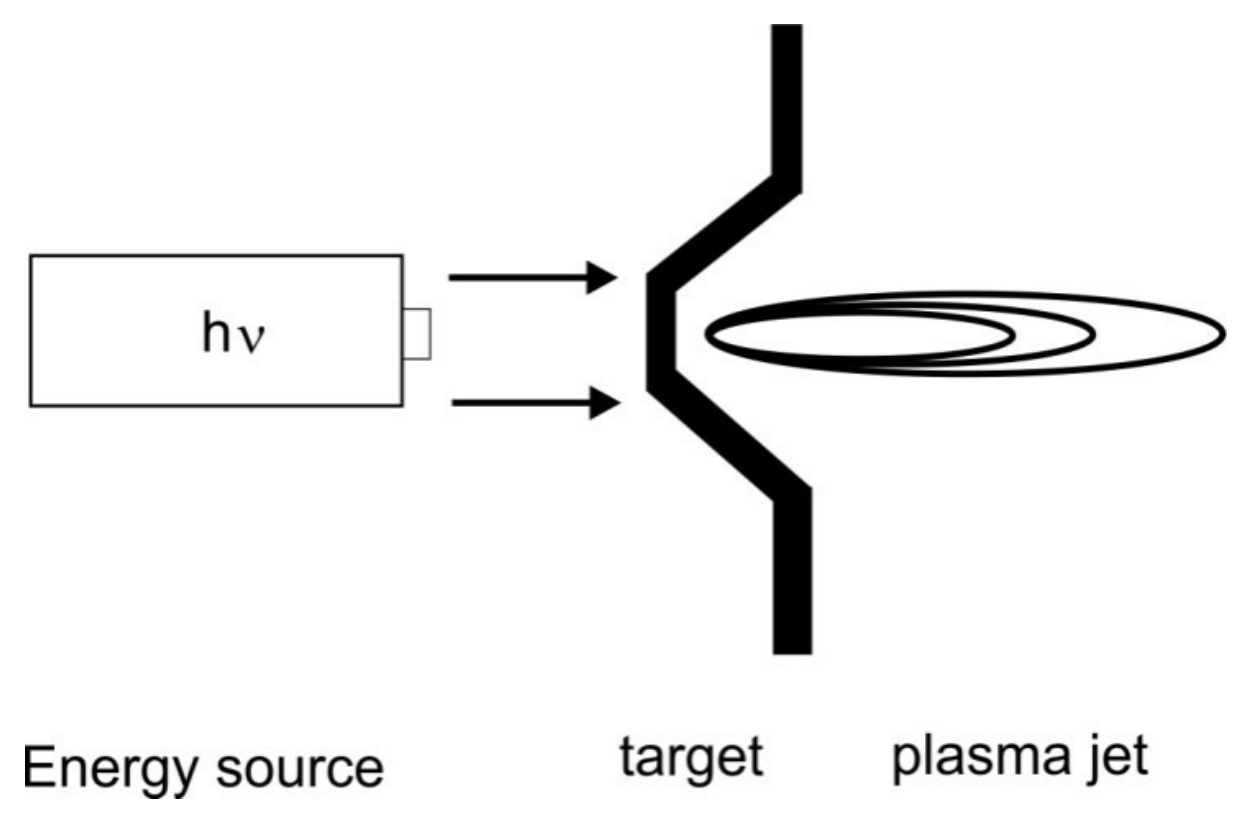

Fig. 5. Configuration of the problem.

A first series of computation experiments (CE) [14-15] was carried out for simulation of the formation of the plasma cumulative jet in the conditions typical of the problems of the laser thermonuclear fusion [16] and astronomical phenomenon [17]. The wide-range semiempirical equation of state was used in simulation for description of the dependence of pressure on density and specific internal energy [14]. The analytical form of final dependencies $p(\rho, \varepsilon)$ for investigated substances permit the effective use of developed EOS in the hydrodynamic computations, providing thermodynamic agreement of the numerical modeling results [14-15].

The results of CE was tested on the experimental results [2] of high speed cumulative jet production by powerful laser pulse for a standard cone target. Two series of CE were carried out. In the first series theprocess of the plasma jet formation was simulated from the "ordinary" conical target of aluminium with the cone angle $90 \mathrm{deg}$. In the second series the target configuration was changed: aflat plate made of cooper was placed in the cone apex and the geometry of the problems was given in the work [2]: the thicknessof the "compressing" plate was 0.8 time the thickness of the coneside wall, the diameter of the smaller base of the conical targetwas 0.3 times the diameter of the greater base.

The target configuration at $1.5 \mathrm{~ns}$ is shown in Fig. 6. The notation: green- the configuration of the cooper plate, blue - the material of the target (the materials of a cumulative jet are in a plasma state caused by the melting of metalin a strong wave and its subsequent expansion). The parameters of the plasma jet are the following: at $1.5 \mathrm{~ns}$ the pressure on the axis is $6400 \mathrm{GPa}$, the speed of the jet is $95 \mathrm{~km} / \mathrm{s}$, at the time moment $1.6 \mathrm{~ns}$ the pressure is $8400 \mathrm{GPa}$, the speed of the jet is $115 \mathrm{~km} / \mathrm{s}$ (Fig. 3). A solid line shown the pressure distribution along the symmetry axis and a dashed line shows distribution of the jet axis speed. The pressure and axial speed distribution at $t=1.5 \mathrm{~ns}$ are shown in the Fig. 7.

The comparison with the parameters of the jet formed from the "ordinary" conical target from CE and experiment [2] showed that the increase of the plasma jet speed in the suggested configuration is $25-30 \%$ and the increase of jet pulse is more than 90 times. 

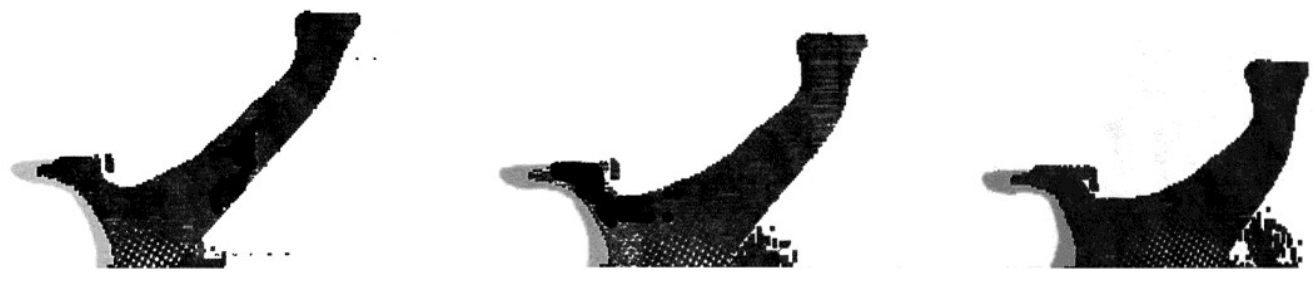

Fig. 6. Dynamics of cumulative plasma jet formation. From left to right: $t=1.357 \mathrm{~ns}, \mathrm{v}=77.41 \mathrm{~km} / \mathrm{s}$; $\mathrm{t}=1.5 \mathrm{~ns}, \mathrm{v}=95.91 \mathrm{~km} / \mathrm{s} ; \mathrm{t}=1.625 \mathrm{~ns}, \mathrm{v}=115.88 \mathrm{~km} / \mathrm{s}$.

64

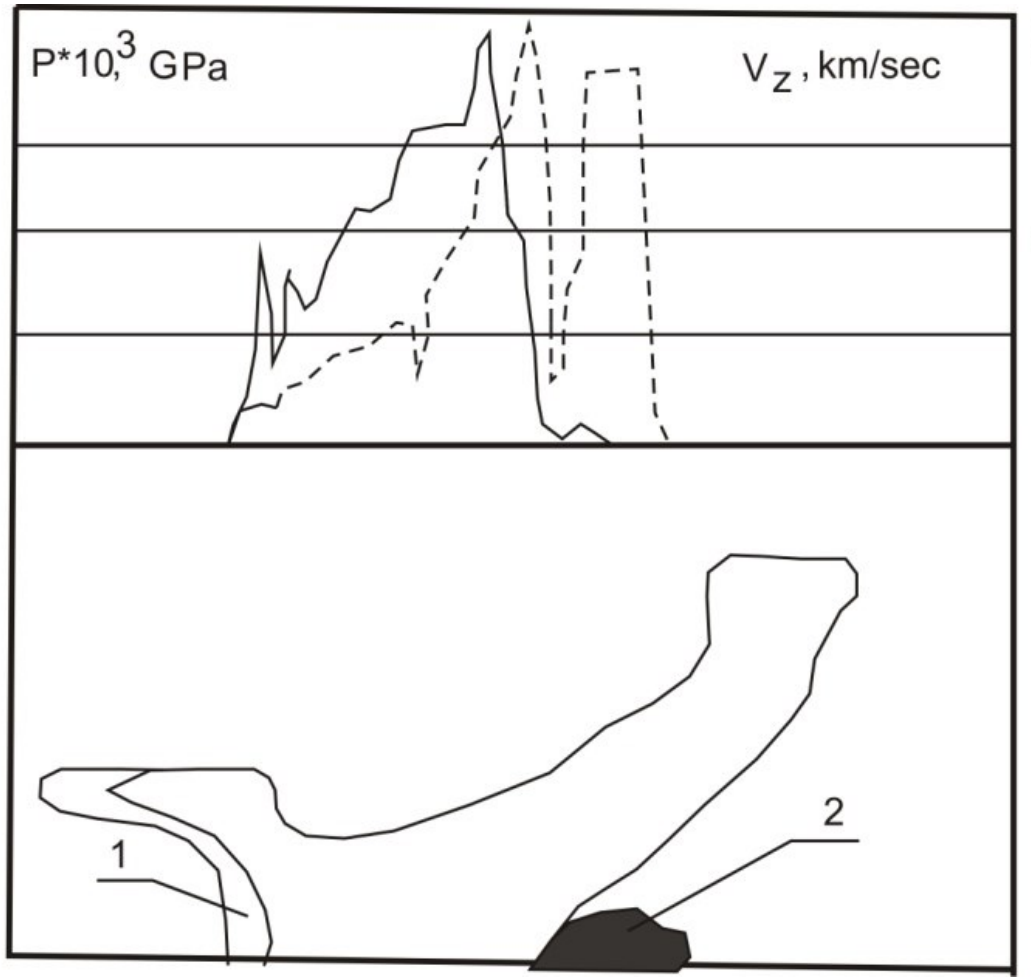

96

48

Fig. 7. Simulation of a cumulative plasma jet at $\mathrm{t}=1.5 \mathrm{~ns}$ : $1-$ cooper plate, $2-$ plasma jet.

\section{CONCLUSIONS}

The number of freedom parameters and the way we use scaling laws are linked to the degree of accuracy we want to access for the astrophysical phenomenon. In hypercumulative regime of jet formation we introduce novel free parameters which allow to control the jet characteristics. Hypercumulative plasma jets formed by hollow cones with flat plate imploded via laser ablation of their outer surfaces were investigated numerically. The velocity, shape, and density of the jets are determinated in calculation experiment. Our investigations have shown that the methods of plasma jet creation based on collapse of a thin conically shaped target in hypercumulative regime are rather promising. We have demonstrated that the realization of the hypercumulative plasma jet formation allows increasing both speed and the 
pulse of the formed jet essentially which are not achievable in the classical cumulation. The jets produced by the method described here can be used to investigate jet propagation into ambient media relevant to laboratory astrophysics, plasma hydrodynamics and for certain fast ignition approaches. Such jets can be used to simulate hydrodynamics of astrophysical jets interacting with stellar or interstellar matter. For example, using the hypercumulative effect there is a possibility of creating plasma jets with parameters very interesting from the Inertial Confinement Fusion and astrophysical point of view. Also this physical principle may be applying to optimization the hydrodynamical mechanism of the flux generation, related with the current sheath compression rate towards the axis [18-19].

Another possible application of hypercumulative plasma jets-generation astrophysicallike plasma jets and accretion columns in plasma of kilo-joule nanosecond laser pulses. This will, in particular, to examine and describe the nature and parameters of the collisional and radiative processes in the plasma at the time of formation and propagation of the plasma jets and accretion columns of the accretion of matter on the surface of stars (white dwarfs).

The perspectives of application of hypercumulative principle of plasma jet formation are, in our opinion, on the possibility of obtaining plasma jets with characteristics that were not previously achievable, not only in the axially symmetric geometry, but in a flat and circular. The proposed principle of formation of plasma jets allows (due to the new free parameter) to carry out optimization of characteristics of the jets.

\section{References}

[1] Anisimov S. I., et al., Pis'maZh. Eksp. Teor. Fiz. 41(N5) (1985) 191-193.

[2] S. P. Nikitin, et al., Physics of plasmas 15 (2008) 050703.

[3] A. Kasperczuk, et al., 35th EPS Conference on Plasma Phys. Hersonissos, 9 - 13 June 2008 ECA Vol.32D, P-1.117 (2008),

[4] C. D. Gregory, et al., Central Laser Facility Annual Report, 2006/2007, pp. 66-69.

[5] Michael A. Purvis, et al., Phys. Rev. E81 (2010) 036408.

[6] Pei Chi Chou, Joseph Carleone, Robert R. Karpp., J. Appl. Phys. 47 (1976).

[7] G. Birkhoff, D. McDougall, E. Pugh, G. Taylor. J. of Appl. Phys. 19 (1948) 563-582.

[8] M. A. Lavrentyev., Achievements of Mathematics 12(4) (1957) 41-56.

[9] V. F. Minin, I. V. Minin, O. V. Minin., International Journal of Modern Applied Physics 2(N3) (2003) 130-141.

[10] V. F. Minin, I. V. Minin, O. V. Minin, et al., "Devices for plasma jet formation" Certificate of authorship 1508938, USSR (1990).

[11] V. Minin, O. V. Minin, Proc. of the Int. Symp. On Intense Dynamics Loading and its Effects, Chengdu, China, June 9-12, 1992, pp. 589-591.

[12] V. F. Minin, O. V. Minin, I. V. Minin. Proc. $11^{\text {TH }}$ Int. conf. on actual problems of electronic instr. Eng. (APEIE) - 30057, 2rd - 4th October, 2012, ISBN: 978-1-46732839-5, V.1, NSTU, Novosibirsk, 2012, pp. 34-52.

[13] V. F. Minin, I. V. Minin, O. V. Minin. Patent of Russia N2412338 (2011). 
[14] V. F. Minin, O. V. Minin, I. V. Minin., Chapter 1 in: Computational Fluid Dynamics Technologies and Applications, ISBN 978-953-307-169-5, publishing date: July 2011. Ed. by I.V.Minin and O.V.Minin. pp.1-28.

http://www.intechopen.com/articles/show/title/calculation-experiment-technology

[15] V. F. Minin, I. V. Minin, O. V. Minin. Proc. of the Int. Symp. on Intense Dynamic Loading and Its Effects.- Chengdu, China, June 9-12, 1992, P. 431-433.

[16] F. Suzuki-Vidal, et al., Journal of Physics: Conference Series 370 (2012) 012002.

[17] V. Gaibler, et al., Mon. Not. R. Astron. Soc., 1-14 (2012).

[18] N. V. Filippov, et al., 1998 ICPP and $25^{\text {th }}$ EPS conf. On contr. Fusion and plasma physics, Praha. ECA vol.22C (1998) 2884-2887.

[19] E. O. Baronova, et al., Plasma Physics Reports 38(9) (2012) 751-760. 